\title{
SEMIÓTICA Y VERDAD: LA REALIDAD DEL SIGNO DESDE LA PSICOLOGÍA DEL DESARROLLO ${ }^{1}$
}

\author{
SEMIOTICS AND TRUTH: \\ THE REALITY OF THE SIGN FROM DEVELOPMENTAL PSYCHOLOGY
}

\author{
Fernando Gabriel RODRÍGUEZ \\ Universidad Argentina de la Empresa (UADE) \\ Instituto de Ciencias Sociales y Disciplinas Proyectuales (INSOD) \\ fgrxyz@gmail.com
}

\begin{abstract}
Resumen: La relación entre significación y verdad ha sido, para algunas tradiciones, una marca definitoria del hecho semiótico. Este planteo vale tanto para abordajes lógicoepistémicos, atentos especialmente a la noción de referencia, como para modelizaciones realizadas desde la noción de lengua saussureana, concentradas en el mecanismo sígnico (respectivamente, referencialistas y significacionistas). Junto con ello, los dos tipos de abordaje, el primero trabajando desde la proposición, el segundo desde las oposiciones del nivel significante, conciben esencialmente la semiosis desde la matriz lingüística, y ambos convergen finalmente en pensar la verdad como un problema de coherencia interna. Se analizan ambas tradiciones, mostrando sus puntos de afinidad y divergencia, y con apoyo en la psicología del desarrollo de la infancia temprana se objeta que el nexo entre procesos de semiosis y de cognición pueda entenderse como originario. En consecuencia, se discute que la semiótica como disciplina deba plantearse límites en términos veritativos o epistémicos.
\end{abstract}

Palabras clave: Verdad. Semiosis. Lenguaje. Ontogénesis.

Abstract: The relationship between meaning and truth has been, for some traditions, a defining mark of the semiotic fact. This is valid both for logical-epistemic approaches, paying special attention to the notion of reference, and for modelizations based on Saussure's notion of langue, centered on the signification mechanism (respectively, referentialists and significationists). Together with this, the two types of approach, the first working from the proposition, the second from the oppositions of the signifying level, essentially conceive the semiosis from the linguistic matrix, and both finally converge in thinking the truth as a problem of internal coherence. Both traditions are analyzed, showing their points of affinity and divergence, and with support in the psychology of early childhood development it is objected that the nexus between semiotic processes and cognition can be understood as original. Consequently, it is discussed that semiotics as a discipline should be delimited by truth or epistemic terms.

Keywords: Truth. Semiosis. Language. Ontogenesis.

\footnotetext{
${ }^{1}$ El presente artículo es parte del proyecto de investigación ACyT P20S52 Representación, imaginación y lenguaje en la práctica histórica de la Universidad Argentina de la Empresa (UADE).
} 


\section{INTRODUCCIÓN}

Aunque con una larga historia, la teoría semiótica (re)nace institucionalmente en los años 60 del pasado siglo (Nöth, 2000). Desde sus comienzos en la antigüedad, de una u otra manera estuvo vinculada con la cognición y la idea de verdad. Esta vinculación supo consolidarse en el Medievo (Pinborg, 1972), obtuvo mayoría de edad con Locke (en cuyo pensamiento se fundió como una de las tres extensas ramas del saber junto a la física y la ética), y luego fue desarrollada hasta el detalle por la ingente obra de Peirce. Pese a la variedad de los procesos de semiosis que abarcaba el modelo peirceano, durante el siglo $\mathrm{XX}$ el parentesco entre signos y cognición estuvo fundamentalmente concentrado en el lenguaje. El objetivo de este texto es evaluar cómo la división semántica entre referencia $\mathrm{y}$ significación fue proseguida y radicalizada por dos tradiciones de manera independientes (una dentro de la filosofía analítica, otra, continental, sobre todo inspirada - pero no exclusivamente - por la moderna lingüística de corte saussureano). Ambas permanecieron, de maneras diferentes, dentro de una discusión en la que la verdad y la semiótica serían polos codependientes. Quienes llegaron a entrever que el signo puede hacer distintas cosas más allá de sus funciones epistémicas no lo pensaron desde el marco general de la palabra y de sus posibilidades. Sólo a partir de la renovación de los estudios sobre el gesto y sobre la ontogénesis de la capacidad semiótica en niños pequeños puede removerse la idea de una conexión de tipo coesencial entre semiosis y conocimiento.

Desde san Agustín a nuestros días la definición canónica del signo describe su mecanismo como un aliquid pro aliquo, algo [que está] por algo [más] (Eco, 1990 [1984]; Nöth, 2000; Vater, 2005). Aquello a lo que el primer aliquid remite se quiebra en la diploplía que Frege inauguró (en rigor, reactualizó) a fines del siglo XIX y que dio origen a la semántica filosófica contemporánea a partir de la discriminación entre la referencia y el significado. Esta duplicidad es lo que este trabajo se dispone a examinar. El primero de estos dos conceptos hizo centro en la lógica y la epistemología para los teóricos de la filosofía analítica ( $\sin$ la postergación de los significados, el único modo de acceder al referente desde la obra seminal de Frege), mientras que el pensamiento orientado a los signos en sí mismos, colocando el foco sobre una semiosis vuelta hacia sus propias pautas de funcionamiento, bosquejó una referencia interna al orden de la significación, de un modo muy alejado de las intenciones saussureanas en las cuales, en buena medida, se amparaba. Estas dos líneas de trabajo prolongaron la ecuación semiosis = cognición de la semiótica en su más genuino y vasto espectro de fenómenos, desconociendo otras variantes sígnicas elementales y determinadas constricciones psicológicas que se encuentran en los cimientos de la significación humana.

En efecto, el pensamiento analítico tendrá su foco en la noción de referencia, incluso para proclamarla inescrutable (Quine, 1960) o para invaginarla en interpretaciones radicales (Davidson, 1973). Aunque nunca podría decirse que hubiera desatendido el plano del significado, es manifiesto en ella, marcada por los afanes de evaluar 
lógicamente la expresión lingüística, que el objetivo es entender la forma en que el significado descubre y encubre el referente. Desde este mirador se han realizado incuestionablemente aportes importantes al significado, pero sin resignar jamás, como horizonte principal, el referente de enunciados o formulaciones proposicionales, problematizados para llevar a la ciencia un instrumento depurado de apoyo o metateoría. Sólo por esto podemos llamar a este particular enfoque referencialista, marcando un contraste con la tradición continental que nace de Saussure y, concentrada en el sistema de la lengua (del que por definición se excluye al referente empírico y a la verdad), únicamente considera la semántica de los significados (por lo que hablaremos de ella como tradición significacionista). Pese a que este exclusivo compromiso con la lengua soslayaba en un comienzo toda discusión sobre el conocimiento, la expansión del saussurismo a otros espacios de investigación (Rodríguez, 2011; Sazbón, 1976) rehabilitó en su seno los debates sobre la verdad, aprisionada en el lenguaje o como texto (para afirmar, en la derivación postestructuralista, que il n'y a pas de hors-texte, según Derrida). De este modo, tanto los referencialistas como, de su lado, los significacionistas (antireferencialistas si hablamos de referentes gnoseológicos) piensan la semiosis como un tipo de proceso psicológico-ontológico esencialmente relacionado con la noción de verdad. En sentido contrario, se argumenta que si, desde luego, saber y semiosis se requieren y apuntalan recíprocamente, no son identificables y, por consecuencia, la semiótica debe emplazarse sobre una distinta base.

En la segunda parte del artículo se despliegan aquellos puntos estratégicos del derrotero referencialista y cómo aquí la referencia conservó siempre el protagonismo, incluso luego de advertir su pecado descripcionista en los inicios. La atención dada al formato proposicional, que implica sin mediaciones al valor veritativo, y el hecho de que, con muy pocas excepciones (Goodman, 1968), en el seno de esta tradición nunca fuera considerada la semiótica en todas sus manifestaciones, sino acotada a los perímetros lingüísticos (y a los usos adultos), pudo haber sujetado su trabajo en torno a los aspectos cognitivos de la significación. En la tercera parte se despliega el movimiento que, de forma inversa, llevó de la lengua a una amplia lingüistización de la cultura y de la realidad. En la cuarta parte, desde la propuesta del segundo Wittgenstein de concebir como uso los significados, se rastrea en la génesis de los usos verbales el tipo de las vivencias que, conforme los estudios de psicología del desarrollo, introducen la significación en la experiencia del niño pequeño sin ninguna participación de la idea de verdad, lo que sería una pauta para destacar cómo la cognición y la semiótica sólo se interfecundan en una segunda etapa ontogenética, y que por ende la semiótica qua disciplina debe concebirse más allá de los fronteras del conocimiento y la verdad. Un recorrido del tipo que proponemos no puede más que tocar puntos neurales de un espacio temporal del que sólo se busca destacar una tendencia luego sometida a discusión. Por ello las citas y las referencias bibliográficas se han escogido, de una cantidad inabarcable, para trazar con ellas una hoja de ruta imprescindible. 


\section{LA TRADICIÓN REFERENCIALISTA}

La distinción referente-significado tiene largos antecedentes. Los estoicos habían separado el semainómenon (significado) y el tynchánon (cosa). Los medievales pensaron por una parte res y signum, la cosa en sí misma y su agente semiótico sustitutivo, de otra parte, al interior del signo, la significatio (que abarcaba por igual la referencia y el significado), la función del signo en la proposición o suppositio (uso o mención, en términos actuales) y la llamada appellatio, evocación de un existente aquí y ahora, ni futuro ni pasado (Beuchot, 1987). En ambos antecedentes la semiosis está vinculada con la cognición, lo que fue confirmado en la modernidad por Locke (2005 [1690]), quien entendía que la semiótica era en definitiva epistemología. Peirce (1972) no dudó en homologar semiótica con lógica. En las postrimerías del siglo XIX, Frege (1892) replanteó en sus términos la distinción de los estoicos en el interés de conformar una lógica pura, capaz de sortear dificultades de las lenguas naturales y operar con pensamientos nudos. Contra el distingo de Mill (1905 [1843]), según quien los nombres no son portadores de significado, para Frege cualquier expresión, completa o incompleta (se supone que los nombres son completos o, técnicamente, saturados, mientras que los predicados son insaturados), posee una Bedeutung (referente) y un significado (Sinn). Los nombres tendrían un referente por sí mismos, pero los predicados solamente por asociación con nombres, de los que recibirían la determinación o concretud, dado que por su condición de relaciones o conceptos no tienen sino la posibilidad de vincular o categorizar. Fuera de las alternativas con las que puede significarse, lo significado es siempre el referente. Toda expresión implica objeto o referente por un medio que lo muestra de una forma u otra, coincidiendo en esto Frege y Peirce, ya que el aspecto era a su vez, en éste, la pauta con la que los distintos signos estaban ligados con su objeto (ground).

Bajo esta inspiración, el joven Wittgenstein iba a plantear, en el contexto del Tractatus (1963 [1922]), que exclusivamente había significado en la expresión informativa, ligada a la triple convergencia entre lenguaje, pensamiento y mundo (una mirada con obvias reverberancias de los modi medievales). Todo lo restante, sin cabida en el debate sobre relaciones de verdad, es a su juicio místico (o ético-metafísico). En esa misma huella de una significación ligada a la verdad y de un lenguaje atado a describir el mundo, el círculo de Viena continuará después algunas de las puntualizaciones tractarianas a la búsqueda de una sintaxis lógica del mundo (Carnap) y de una ciencia instituida desde el principio de verificación.

En paralelo con esta pesquisa sintactista y lógica centrada en los parámetros formales, el pensamiento del lenguaje de corte analítico fue dibujando un giro que condujo hasta la reivindicación del lenguaje ordinario. Frente a la búsqueda de un idioma formal perfecto y sin ambigüedades, John L. Austin notó que las lenguas naturales son lo suficientemente ricas como para permitirnos tratar con el mundo real, ya que, bajo sus trampas evidentes (por ejemplo, homologar los enunciados de predicación e identidad), poseen todas las 
categorías fundamentales para que los hombres se entiendan unos con otros respecto de su experiencia vivencial. "Nuestro común stock de palabras incorpora todas las distinciones que los hombres han hallado conveniente hacer, y las conexiones que han hallado conveniente establecer, durante la vida de muchas generaciones" (Austin, 1975 [1962]: 174). De modo que la experiencia se halla recogida en la sabiduría de nuestra lengua cotidiana, con cuya aptitud para nombrar objetos y acontecimientos reaparece la verdad, aunque no sea aquella verdad científica o propia de la razón más depurada. En este punto, si el lenguaje habla del mundo con verdad o falsedad, es por la referencia, según había puesto de relieve Strawson (1950). En el espíritu del distingo peirceano entre sinsigno y legisigno, Strawson marcaba que a una expresión in abstracto (type) no corresponde valor de verdad, porque esta surge cuando el signo se aplica a una circunstancia (sign-token). Esta situacionalidad de la expresión es lo que permite a su vez a H.P. Grice (1975), cuya obra gira centralmente en torno a la conversación (principio de cooperación, significado intencional, implicaturas), postular una máxima de relevancia (Sea pertinente en lo que diga) respecto de un marco neutro u objetivo que permitirá evaluar la pertinencia de lo dicho (marco que implica referencia al mundo).

La referencia como tal incluso había llevado a su cuestionamiento. La transitada viñeta de Quine (1960) donde un lingüista debe descifrar lo que el hablante de una lengua pretende comunicar cuando, a la vista de un conejo, dice "Gavagai". De acuerdo con Quine, los datos perceptuales no permiten decidir si "Gavagai" se refiere a conejo, a la conejidad, a estadios de conejo, etc., y la tendencia a pensar que el hablante alude al animal conejo es nada más un sesgo que acomoda el hecho al trasfondo lingüístico de ese lingüista occidental (background language). Para nuestro objetivo, poco puede esperarse de considerar si Quine acaso descuidó variables psicológicas fundamentales (la atención, las leyes perceptuales estudiadas ya por la Geltalttheorie, o las hipótesis actuales sobre adquisición de los significados de palabra — restricciones de mutua exclusión verbal, correspondencia rápida entre cosas y designaciones novedosas, propensión a utilizar estas designaciones prima facie en objetos totales más que para partes o características, apoyo en categorías o taxa prexistentes (cf. Karmiloff \& Karmiloff-Smith, 2001) - , o de redargüir que acaso esa inescrutabilidad puede también, con el mismo criterio, pretenderse al interior de cada lengua y, sobre todo, cuando el niño debe segmentar la cadena del habla del adulto y aparear sonidos con significados. Lo que sí nos concierne es cómo, bajo esta hipotética inescrutabilidad, la referencia, fijada por el recurso a la conducta y a "puntos de referencia no verbales, circunstancias no verbales que se puedan apreciar intersubjetivamente y asociar inmediatamente con la emisión adecuada" (Quine, 1988 [1974]: 53), continúa siendo problemática (Ávila Cañamares, 2014; Campbell 2002).

La referencia inescrutable no libera de tener que rendir cuentas de cómo es posible llegar a entendernos sobre el mundo o sobre lo que suponemos, damos por sentado o asumimos que sea el mundo. Así llegamos a la interpretación radical de Donald Davidson (1973), para quien la persona acepta en calidad de verdaderas ciertas expresiones no por 
la correspondencia entre los contenidos proposicionales o de creencia o, muchos menos, expresiones nominales y entes identificables por artes de la palabra, sino por la garantía de crédito que arma holísticamente la red de expresiones de una lengua para una comunidad. Es el conjunto de significados lo que la comunidad acepta como verdadero, y esta verdad reticular se afirma y se sostiene de la trama de sus elementos, de forma que los que rompen la coherencia o la amenazan encarnan la falsedad. Por supuesto, los nexos causales entre los fenómenos de entorno y las acciones del sujeto impiden que la verdad bajo las significaciones sea una pura discursividad; lo verdadero, o el conjunto de las creencias, creencias verdaderas, se encuentra ligado a las conductas cotidianas compartidas y exitosas. Conforme con ello, la teoría de Davidson sobre el significado y su aptitud referidora incluye un tratamiento de las creencias de los interlocutores, que a la vez no pueden ser establecidas sino desde la interpretación de lo expresado por los mismos interlocutores, de forma que la interpretación de la conducta de éstos, por ende también su concepción del mundo, y la decodificación de su lenguaje desembocan en una única interpretación que es bien denominada radical. Lo que alguna interpretación particular asuma como verdadero debe ser coherente con las interpretaciones en las que el resto de los hablantes estarían dispuestos a asentir a una oración. Mucho de este punto de vista reaparece en la evidencia empírica que la psicología pone a disposición para entender el acceso del niño a su lengua materna (ver sección 4), pero por concentrarse en las interacciones desatiende los casos de significación que no implican directamente el respaldo de los demás (aunque esto debe suponerse, desde luego, indefectible e indirectamente como cimiento y aval de toda realidad).

Como quiera que sea, la referencia empírica y la relación con la verdad de una semiótica forjada desde la proposición (por mucho y muy claramente que visibilice los problemas de los nombres como agentes de individuación) es la característica del enfoque analítico-epistemológico. Como contrapartida, los estudios del significado en esta línea de investigación apenas si se ocuparon de los signos no verbales (hay menciones, por ejemplo, en Quine, a la ostensión, o la conducta en Davidson y en Austin, pero para indicar cómo tropiezan con el mismo obstáculo que la palabra). La incontestable realidad ontogenética de que el lenguaje no es la primera semiosis del sujeto no fue incorporada seriamente a la filosofía analítica. El afán epistémico había señalado un rumbo diferente, responsable de otro olvido no más disculpable, aunque advertido a tiempo: aquel de las funciones del lenguaje no afectadas al conocimiento. Será entre las minuciosas investigaciones de Austin sobre la performatividad y la triplicidad del acto de habla (Cf. sección 3) y antes en Wittgenstein (1953) donde el concepto de significado sea llevado a una pragmática fundacional. En las Investigaciones filosóficas se lee el pasaje célebre sobre el uso-significado.

[P]ara una gran clase de casos de utilización de la palabra "significado" — aunque no para todos los casos de su utilización (itálicas originales) — se puede explicar esta palabra así: el significado de una palabra es su uso en el lenguaje. Y el significado de un nombre se explica a veces señalando a su portador (1988 [1953]: 43). 
Resulta con ello que habría dos formas elementales de la significación, una ostensiva, otra de clase ejecutiva, ambas dentro de una pragmática fuente de toda semanticidad. La aclaración respecto de los nombres es, al mismo tiempo, una valiosa concesión a la versión agustiniana del aprendizaje de palabras, criticada antes en $\$ 1$ y ahora revitalizada al interior de una visión más amplia. Gesto y actividad, en consecuencia, serían formas subyacentes al lenguaje y al conocimiento.

Todo ello se condice con el tratamiento hecho por Wittgenstein de la certeza (1969), una instancia social precognitiva, preveritativa, hecha de convicciones básicas sin evidencia ni argumentación, sólo certificadas desde la eficacia de nuestras acciones en el mundo circundante. Estas certezas forman un sistema que puede ser sometido a duda por cada unidad o afirmación independiente, pero no en su completud, porque no hay otros asideros con los que emplazar algún cuestionamiento. Cuando el saber científico pone alguna certeza contra la pared, este no ha renunciado a las categorías más generales del primer sistema de articulación sujeto-mundo, esto es, al punto de partida de donde le es permitido cuestionar. No son verdades epistémicas, porque no tienen un reverso (posibilidad de falsedad), sino seguridades prácticas surgidas de la cotidianeidad y confirmadas por la acción frustrada o exitosa. El parecido con el esquema ulterior davidsoneano es, en lo básico, patente. Este nivel irrebasable de continuidad o nexo con las cosas, dado por las asunciones y no por razones, se verifica en la ontogénesis, donde la significación no es todavía conocimiento, no tiene siquiera forma proposicional y, sin embargo, brinda al niño, incluso antes del habla, una herramienta inestimable para enriquecer su interacción con otros e ingresar al mundo cultural.

Quizás el problema estuvo en pensar el lenguaje desde el discurso epistémico y para el lenguaje adulto, esto es, para el hablante competente. La filosofía analítica vio la falacia llamada descripcionista y amplió las funciones del lenguaje, pero no bastó identificarla para pensar al lenguaje como un emergente de alguna semiótica anterior y más profunda. Las funciones fática, expresiva y conativa de los signos del lenguaje son continuaciones de los usos de otros signos previos (Rodríguez y Español, 2019), y la aparición del lazo significación-verdad lleva a olvidar el origen no cognitivo de toda semiosis. Pragmática antes que semántica o sintaxis.

\section{LA TRADICIÓN SIGNIFICACIONISTA}

Dentro de los proyectos de interpretación semiótica de la cultura, en el pasado siglo hubo abordajes que expandían la significación sobre ámbitos que — en otro tiempo — se habrían considerado ajenos a su competencia, y a la vez ensayos antitéticos, asemantizadores, que reducían a estricta formalización los meollos y resortes de la fórmula aliquid pro aliquo. Dentro de los primeros, algunos autores como Lotman y Eco convirtieron la semiosis en el elemento mancomunador de todos los fenómenos de la cultura. La significación opera, según Eco, como un encadenamiento de interpretaciones 
sucesivas, sin comienzo ni espernada, que la condición de interpretante-interpretable de los signos relanza hasta el infinito (no hace falta señalar el trasfondo peirceano de esta tesis). En la vereda opuesta, el estructuralismo de cuño francés elaboró un formato básico de inspiración lingüística muy alejado de este esquema y sólo afín en la deriva de la significación. Si comparte etiqueta con una semiótica semanticista-cultural no es porque defendiera alguna preeminencia del significado, sino debido a una postergación del referente empírico-epistémico (aunque si en un comienzo este era marginado en la obra de Saussure, volvió más tarde a reclamar sus fueros de la mano del postestructuralismo). Para desbaratar cualquier enredo, digamos que el pensamiento referencialista nunca ha renegado de la faz semántica del signo, mientras que los significacionistas soslayaron a los referentes en cuanto entidades del mundo exterior. Ambos agrupamientos significacionistas se distinguen en torno de la actitud respecto de la referencia: esta es, por una parte, indiferente a la expresión verbal o de otra clase; se encuentra, por otra, sometida a lo que las palabras y otras formas significativas pueden modelar como lo referido.

Los significacionistas-culturologistas (en acepción lotmaniana) nunca renegaron de las relaciones íntimas entre semiótica y verdad. Eco lista entre los procesos de semiosis a la percepción, que es cognición e implica una variante singular del acto inferencial (1999 [1997]). La pertinencia de entender la percepción como un proceso cognitivo asimilable a un ejercicio de semiosis quedará, por motivos de espacio, para otra oportunidad. Hemos de concentrarnos sobre la línea estructuralista, porque en ella la conexión entre signo y verdad vuelve a poner el centro en el lenguaje, lo que permite una comparación con la postura referencialista, pero vaciándolo de significación y convirtiendo la semiosis en una en una abstracción que sólo en segunda instancia produce un nivel semántico y este, a su vez, un nicho para la verdad (sintaxis pura $\rightarrow$ semanticidad $\rightarrow$ epistemología).

Si el movimiento de Saussure había implicado ir del lenguaje hacia la lengua, ésta un sistema - o estructura - donde el signo es un compuesto de dos subsistemas de unidades oponibles (Saussure, 1916), la fonología y sus desarrollos por el Círculo de Praga, retomados por la etnología lévi-straussiana, subordinarían la esfera del significado a la articulación de los significantes, devenidos insignificantes. La asemanticidad de los fonemas fue el modelo de la danza de elementos neutros con los que la etnología tematizaba el parentesco, la mitología y distintas prácticas sociales. Lo simbólico fue el nombre del registro en el que un orden de unidades desprovistas de sentido, por un mecanismo interno de tipo autogenerado, habilitaba posibilidades tanto como imposibilidades de combinación, forjando de esta suerte una legalidad presignificativa (Barthes, 1967; Lacan, 1966). Lo simbólico insignificante era planteado como aquel registro instituyente de la realidad, el Orden que acoge en su interior toda otra lógica o dinámica, el principio organizador-sujetador del individuo que solo concibe el mundo desde la pauta algorítmica de las combinaciones y se comprende a sí mismo como sujetado. Lo simbólico es lenguaje en un estado puro. Para el estructuralismo, la cultura (que incluye nuestra visión del mundo fáctico o real) respeta el formato de una lengua, 
del sistema opositivo, pero es una lengua reducida a la capacidad autogestiva de unos componentes mudos. Lengua elevada a ser lenguaje (algo contrario al credo saussureano) y reducida a su mínimo histórico. No cuesta percibir la afinidad con los idiomas informáticos, que, en su nivel rudimentario, aquel llamado de lenguaje máquina, no es otra cosa que una oposición entre paso/bloqueo de la corriente eléctrica (lo que Eco iba a considerar, en Segno, 1980 [1973], como el límite inferior de la semiosis o S-código).

La subordinación del mundo real bajo el imperio del lenguaje estaba comprehendida ya en Saussure ("No hay ideas preestablecidas y nada es distinto antes de la aparición de la lengua", Saussure, 1995 [1916]: 155), pero la lengua era infaliblemente una entidad bifronte. Al perder el contacto con el mundo (pues la lingüística no tenía compromisos ontológicos de base), la lengua forjaba sus propios conceptos. De esta manera, las imágenes del mundo captadas por la estructura de la lengua anulaban la referencia en cuanto término epistémico que trascendía al signo lingüístico. Las reglas del nivel significante imaginarizaban lo real. La autonomía de los significantes implica que estos no necesitan respetar características del ente perceptual-factual para labrar su propia normatividad y, de regreso de esta libertad fundacional, disciplinar el mundo ante nosotros. El corolario es evidente: todo es lenguaje y de las reglas forjadas de esta manera hay mundo o referencia en un segundo tiempo. Se derrumba el concepto de correspondencia en un sentido estricto (con algo exterior) y la verdad es siempre una secuela. Un kantismo algebraico ha reemplazado la interioridad de las categorías o los conceptos puros.

Será un autor posestructuralista, Derrida, quien en su crítica de Husserl "La voz y el fenómeno" (1967a) explicite que lo real no es mudo, neutro u objetivo, sino que está ya configurado. Por sobre cualquier esencia del tipo que había inspirado la pesquisa husserliana nuestro acceso al mundo y a las cosas se encuentra mediado, en el origen mismo, por una estructura, aquélla del lenguaje. El sentido de los fenómenos es un efecto de estructura, la cual por su parte está en constante movimiento (mutabilidad e inmutabilidad del signo saussureano). El lenguaje no adviene entonces sobre una experiencia previa, límpida, para nombrar lo que ya es pre-sentido antes de su llegada, sino que en toda experiencia hecha del mundo, el mundo como tal habla al sujeto en el idioma más fundamental, primario, que precisamente en su primariedad oculta la voz del fenómeno. La voz es en verdad lenguaje. Las categorías de comprensión, una estructura o escritura. Contra el fonocentrismo, que tan graves consecuencias habría deparado al pensamiento occidental, una escritura o gramatología (1967b). Frente al acceso a lo esencial del mundo en cuanto realidad genuinamente trascendente, la apuesta de Derrida es no tanto la inescrutabilidad (como en los referencialistas Quine y Davidson) sino una referencia con la forma de una diferencia responsable de su condición. Y cada configuración es susceptible de reconfiguración, porque lo real, y la verdad, quedan localizados dentro de un espacio de trascendental diferimiento: el mundo, en su sentido, se halla desplazado del mundo presunto y accesible para la razón o el Logos. El mundo es escritura que se halla en constante reescritura. Hay que des-construir los quistes y 
endurecimientos de la concepción fonocentrista que, desde Platón, confiaba en la palabra hablada como puente a las Ideas eternas y de este registro inteligible al saber de las cosas. Derribar ideas, categorías, verdades: no hay nada sólido, sino puro diferimientodifférance (1967c).

En Derrida, la estructura supone una verdad a la deriva al interior de una versión de lengua elevada a lenguaje y éste a semioticidad en su radio más vasto. La cuestión epistémica, desalojada por Saussure en su acto fundador (la lengua no tendría que ver con los conceptos de la realidad o la verdad: de hecho "es mal método partir de las palabras para definir las cosas", Saussure, 1995 [1916]: 31), conseguía un salvoconducto para reingresar en cuanto el sistema o la estructura se extendía a todo el perímetro de la cultura y de la racionalidad (pues la razón había pasado a ser sólo una de las formas de la simbolización). Pero el regreso debía respetar la salvedad de que dentro de lo simbólico todo queda subordinado a su juego arbitrario. El objeto es opaco, está perdido entre las diferencias que fragmentan la continuidad del mundo físico en signos discretos. Por este expediente se ponía al discurso oral, equivocadamente valorado por encima de la letra muerta de los textos, en el mismo nivel de opacidad que la escritura. Todo significante está igualmente preso en el equívoco y en la interpretación, por lo que en consecuencia los significados no serían asociaciones firmes con sonidos o soportes físicos sino una emanación del texto o el discurso en el que están dispuestos.

Greimas y Courtés, con un vocabulario menos filosófico, pero bajo la misma inspiración, lo ponen de este modo: la correspondencia entre el mundo del signo y un mundo referencial extrasemiótico no implica más que un nexo intersemiótico. Suscribiendo la tesis de Whorf, la naturaleza no es una referencia neutra sino culturalizada ("las etnotaxonomías dan 'visiones del mundo' diferentes" - Greimas y Courtés, 1993: 312, mi traducción-), por lo que el problema se traduce en la correlación de dos semióticas: una semiótica de percepciones y hechos naturales o macrosemiótica, y un plano cultural que re-ingurgita esta primera en una multiplicidad de versiones posibles. Como se trata de coherencia entre dos órdenes que se co-pertencen, una verdad en el sentido referencialista es imposible. La verdad de ese tipo se ha trocado en la veridicción.

[A]l postular la autonomía, el carácter inmanente de todo lenguaje y, al mismo tiempo, la imposibilidad de recurrir a un referente externo, la teoría saussureana [que en realidad, hay que decirlo, habla de lengua] constriñó la semiótica a inscribir en sus preocupaciones no el problema de la verdad, sino el del decir-verdadero, el de la veridicción (Greimas y Courtés, 1993:417, mi traducción).

Si con el estructuralismo hay una lingüistización (de lenguaje degenerado) para el universo humano (tomando con pinzas la adjetivación "humano", dado que, como apuntara Lévi-Strauss [1970: 357], se trata de disolver al hombre), rechazando una verdad que no fuera veridicción, y si por otra parte los autores referencialistas llegaron a cuestionar la posibilidad de acceso a un referente extralingüístico, lo que sugiere que estas dos líneas argumentales, reñidas en muy distintos frentes, parecen al cabo coincidir, ¿debe 
reconocerse en este punto, por mor de la concurrencia, un mayor crédito a la idea de fondo, al nexo entre semiótica, lenguaje y cognición?

¿A qué ganancia hablar de una estructura-lenguaje-escritura que sólo retiene algunos rasgos del lenguaje en su acepción corriente, rasgos que serían seleccionados, si no caprichosamente, por lo menos bajo un criterio opinable? ¿Las formas lingüísticas resumen toda la semiosis, o habrá que entender que es ésta la que contiene al lenguaje como un derivado, y que la oposicionalidad tiene vigencia solamente en la jurisdicción de las palabras? Una manera de salvar esta polémica, profusamente discutida en la palestra de los argumentos, conduce a las investigaciones longitudinales y/o transversales de extracción empírica que siguen en detalle cómo el niño ingresa al territorio de la significación. Es el camino que indirectamente ha señalado Wittgenstein al poner el acento sobre el uso. Veamos los usos primigenios de los signos que emplean los infantes y evaluemos si cabe asignar a una estructura por oposiciones o a un distinto mecanismo las primicias de la semioticidad. Veamos si en esta instancia el uso está al servicio de la cognición, y si desde los resultados en psicología del desarrollo es posible abonar una disputa que entre estudios culturales y filosofía no ha conseguido resolverse. En la ontogénesis veremos si verdad, conocimiento, estructura y proposición son imprescindibles para establecer una definición de signo que no desentone con su núcleo (y de donde sería también no menos esperable una semiótica redefinida). Antes de entrar en tema, adelantemos que el trabajo de la observación en la infancia temprana revela que el signo, y por lo tanto la semiosis, no surge en el niño por contraste de unidades insignificantes sino por desprendimiento de acciones y de rutinas habituales (hábitos: palabra clave en la teoría semiótica peirceana de la madurez —cf. Short, 2007—). El estructuralista podría señalar, antes de renunciar a su receta, que en ello no hay todavía lenguaje. Pero habría signos genuinos, con significado, que anticiparían la entrada en el Orden simbólico, lo que constituye — como mínimo — un contrasentido.

\section{ONTOGÉNESIS DE LA SEMIOSIS}

Las interacciones neonatales no son significativas del lado de los bebés. Los adultos, por supuesto, las cargan involuntariamente de sentido, pero ello no supone que inmediatamente el niño ingrese a la constelación semiótica, sino que hay las condiciones para que el encuentro con el signo se produzca. Los bebés tienen medios inespecíficos de indicar sus necesidades. Durante las primeras dos semanas tras el nacimiento se identificaron tres clases de llanto: de hambre, cólera y dolor, y luego un cuarto llanto de atención, que posee la particularidad de reclamar por la presencia del adulto por fuera de las necesidades corporales (Wolff, 1987). Además del llanto, la modalidad oral de la expresión permite el sostenimiento de conductas preverbales y presignificativas que no obstante portan carga emocional y allanan la con-sonancia con el semejante por placer, por el gusto de establecer una comunidad de estados afectivos. Pero estas relaciones no pueden tomarse como procesos semióticos auténticos sin adulteración o forzamiento. Los 
juegos de dar-tomar no son más que una praxia. El movimiento del adulto es la señal para que el niño contribuya a la continuidad del ciclo.

En el trayecto hacia la significación es importante distinguir aquí el antecedente protoconversacional (Trevarthen, 1980). A partir de los dos primeros meses, el niño protagoniza un ejercicio lúdico de vocalizaciones alternadas con el interlocutor, sonidos emitidos respetando turnos como en situación de plática genuina, sólo que sin mensaje o contenido. Es un dispositivo armado para compartir con otro, y que aporta el patrón de los futuros diálogos semióticos. Bebé y adulto gozan de un entendimiento mutuo en el que, en puridad, no hay nada que entender, sólo empatía que se prolonga en el deleite por sintonizar, lograr el mismo tono o vibración emocional (Español, 2014). No hay cognición ni signo para decodificar (si la definición de algo por algo, la sustitución de un ente por otro dotado con capacidad para evocarlo, ha de tomarse en serio). Los movimientos del adulto a los que el niño puede replicar con su propio caudal de movimiento no están en las veces de otra cosa: son, en cada caso, indicio desencadenante de una práctica ritualizada. Es una discusión de otro lugar si estos indicios forman parte del mundo semiótico o quedan por fuera (respectivamente, Peirce y Savigny). En lo que nos concierne, la respuesta es indistinta para hablar de significación.

Los intercambios diádicos emocionales asemánticos son prueba de que el niño puede anticipar, en los estímulos que reconoce, las rutinas consecuentes a las que están asociados. Así, guarda silencio hasta que el semejante hace su parte, y en ello se advierte cómo ha incorporado que la situación se encuentra organizada alrededor de turnos. De una manera similar, sabe que luego de comer sigue el momento de dormir, o que cuando se ensucia alguien procede al cambio de pañal. La primera sección de estas rutinas funciona como inductor-señal de lo que todavía no ocurre, pero se prevé. La totalidad de la rutina oficia como plataforma de donde los hábitos desprenden los signos inaugurales (Nelson, 1996). Toda experiencia se fragmenta en eslabones que abren a la posibilidad de asociaciones. Si un primer eslabón cumple la comisión de remitir fuera de sí (y ha resignado su entidad en aras de esa funcionalidad), y si además el tal ligamen no es idiosincrático, sino social (Eco, 1980 [1973]), entonces tenemos un proceso de semiosis, ya que el significante (o el representamen) se ofrece como recurso o medio interpretable para acceder más allá.

La comprensión del signo (de algo como signo) se enriquece cuando la intersubjetividad primaria, caracterizada por esa protoconversación descripta, nutre el formato de la interacción diádica con el objeto, haciendo los enlaces triangulares. Luego de la etapa inicial, cuando el neonato se entrega exclusivamente a relaciones intersubjetivas, y de —más tarde - una incipiente exploración de objetos, el niño consigue vincular las dos mitades de su mundo en la experiencia de atención conjunta, decodificando la mirada de los semejantes como predisposición o intencionalidad sobre las cosas. Siguiendo el vector de la mirada o de la orientación del cuerpo, que decodifica como un pointing, como pista de inminente acción sobre un objeto-referente, la atención del niño se acopla a la del adulto y por este expediente comparte con él un interés 
particular (Escudero-Sanz, Carranza-Carnicero y Huéscar-Hernández, 2013). Todo ello es correlato de haber entendido al otro como un ser intencional y desiderativo volcado a las cosas, y cuando los semejantes se revelan como entes con intenciones y, de forma paralela, se han desarrollado habilidades de emisión oral o gesticulativa suficientemente finas como para emplear, hacia el final del primer año, primeras palabras y signos manuales, se ha cumplido con las exigencias psicológicas para llegar a ser un agente semiótico a cabalidad, esto es, para no simplemente decodificar, sino además para expresar sígnicamente lo que quiera compartirse. El formato inicial de las protoconversaciones se ve entonces fecundado por la competencia para usar los signos, y hacerlo sobre un mundo en común.

Desde un ángulo determinado se podría afirmar que, cuando hay la destreza para nombrar cosas, se abre paso juntamente la equivocación, y el niño puede llamar "perro" a los caballos, con lo que se infiltra en la semiosis la idea de verdad. Pero sería excesivo pretender, antes de que haya una predicación enunciativa o intercambios claramente informativos (que no deben confundirse con usos declarativos, ya demostrativos, ya nominativos - Rodríguez y Español, 2019-), que existiera para el niño una variable verdadero/falso en sus errores de designación. Sólo tenemos, de momento, el precedente ejecutivo acierto/error, mientras que la verdad supone anticipar que un pensamiento pueda estar equivocado (no hay todavía aquella distancia necesaria para pensar sobre el pensamiento y, de antemano, especular en torno de su contenido). Las rectificaciones del adulto establecen el lecho para la futura noción de verdad, pero en rigor, con el aval de estar en una etapa ontogenética marcada por la acción y por procesos de semiosis todavía rudimentarios (caracterizados por su dimensión pragmática), podemos decir que todavía no hay signos vinculados al conocimiento teórico, verdad o falsedad, sino utilitarismo, instrumentalidad, la voluntad de hacerse comprender. El precedente no veritativo de la comunicación había sido reconocido en "Sobre la interpretación”, donde Aristóteles supo identificar las dos utilidades decisivas del lenguaje, la nominación y la predicación, que respectivamente entrañan la categorización y el juicio sobre los entes del mundo (la segunda presupone la primera), pero en la modernidad la prioridad concedida a la lógica olvidó o disminuyó el papel del nombre y, sobre todo, del nombrar, acto de inestimable peso en el progreso de la inteligencia. Incluso al considerar que la semiosis intuitiva de los niños pregramaticales se halla, indefectiblemente, coordinada con objetos inmediatos, la idea de verdad no puede todavía hacerse lugar.

El uso de los signos, en cuyo arco de variantes el nombrar es un peldaño inflexional, se corrobora desde la investigación experta como horma o patrón con el que el niño pondera las prestaciones que estas herramientas le ponen delante. Este contexto primordial no está compuesto por verdades sino por certezas (de extracción wittgensteineana), las cuales, a su debido tiempo, dejarán surgir la duda. Este nivel, antepredicativo y habitado por objetos al alcance de la mano, constituye el pedernal de la ulterior vacilación y de los planteamientos epistémicos en el sujeto. 
El universo de certezas socialmente elaboradas proporciona al signo empleado su confirmación. No es un aprendizaje que involucre a la verdad, sino una actividad correcta o incorrecta. De allí que toda cognición en términos veritativo-proposicionales sea un $a$ posteriori montado a la espalda de procesos psicológico-semióticos, que sólo ocurrirá cuando tenga lugar el cruce entre la significación y el pensamiento reflexivo. El signo inaugural, si no es del tipo verdadero/falso, responde a funciones anteriores: fática, declarativa, apelativa (la declarativa no es informativa — Rodríguez y Español, 2019-). Lo que evidencian los estudios de infancia temprana es que la prescripción griceana de un principio de cooperación existe en comunicaciones sin palabra y antes de que haya un mensaje para decodificar, y que, cuando hay mensaje, este es primero un elemento sígnico al servicio de la interacción y de la acción con cosas y sólo más tarde un aparejo del conocimiento. Somos seres sociales antes que semióticos y que epistémicos; es condición de tipo necesario que seamos sociales y semióticos para poder después lanzarnos a la búsqueda de la verdad.

Una segunda forma de verdad, la que se opone a la mentira, tampoco puede plantearse en el comienzo del uso de signos. Ella depende de una inteligencia capaz de sacar ventajas de otros individuos, lo que es una habilidad que implica lectura de mentes y que el niño no consigue capitalizar en beneficio propio sino desde los 3 años (para otros especialistas, desde los 2 años —Leduc, Williams, Garibello y Talwar, 2016-). Para mentir es necesario un cálculo de situación. Cuando un niño rompe el jarrón y le preguntan quién ha sido, confiesa inocentemente hasta que aprende el modo de evitar las consecuencias. Con el correr del tiempo advertirá cuántos recursos tiene en la palabra (también en el gesto) para desviar la sospecha en otra dirección. Pero sucede, en cualquier caso, que un primer nivel de significación basal debe ser manejado para prestarse a intenciones de este tipo. Sin los significados léxicos no se puede mentir ni hablar con la verdad, de forma que también esta verdad, adjunta de la honestidad, no es el modo inicial de la semiosis. La mentira requiere de un significado literal para efectuar con él una maniobra interesada. Por consiguiente, las dos formas de faltar a la verdad, por equivocación y engaño, se hallan emplazadas sobre un escalón de acción semiótica eficaz. Y de las dos, solo el mentir rompe el principio de cooperación. Las dos verdades moral y epistémica no son parte de la semiosis sino como empresas derivadas que persiguen sus particulares objetivos en hombros del signo y sus potencias.

En conexión con este punto no es gratuito volver a la posición de Derrida. Como es sabido, su polémica con Searle se entabló alrededor de la lectura de Austin. Mientras que para Austin y Searle (1969) hay un nivel de usos lingüísticos primario y otro (que incluye ironía, doble sentido, cita, oratio obliqua, etc.) de carácter parasitario, para el francoargelino no existe la división primario/secundario (un habla intencional y comunicativa que logre satisfacer las pretensiones de una transmisión, versus un habla que persiga su objetivo de forma sesgada o por elevación), ni algo que transmitir que sea en sí mismo o de lo que haya una experiencia pura (Navarro Reyes, 2010). En la filosofía de Derrida no hay puntos de contacto con un mundo por detrás de los significados: ni acción ni 
percepción, ninguna vía es directa, ambas se encuentran perfiladas por la diseminación de los sentidos y de la interpretación, lo que transforma al mundo en texto y en perpetuo diferirse de sí mismo (de nuevo: il n'y a pas de hors-texte). Para este esquema, los actos lingüísticos llamados insinceros serían coesenciales al lenguaje y este, por su lado, comprimido como una estructura de elementos contrastantes, sería por su parte artífice de la fragmentación de lo real.

Si hay, en efecto, un primer escenario de expresiones de sentido irreductible y otras que las presuponen o, por el contrario, si actos comunicativos plenos o exitosos tanto como fracasados son variantes codeterminadas es, de nuevo, algo que sólo puede dirimirse en la ontogénesis. Cuando el niño utiliza una palabra conocida, la ha escogido de su repertorio precodificado. El código fonemas-significación se consolida, como vimos, a partir de las interacciones, de las cuales en determinada instancia logra emanciparse en calidad de léxico mental. Las significaciones básicas pueden cambiarse en el contexto, cuestión que daría un sostén para el enfoque derrideano, pues la interpretación de los significados queda entonces ligada a factores que exceden y quiebran cualquier hipotético ligamen férreo. Pero el significado ocasional, que puede ir inclusive contra la acepción estándar, depende en principio de las convenciones con las que él mismo pone distancia. La interpretación implica siempre claves interpretativas, de modo que, en el intento por deconstruir, y mostrar que el sentido no puede aferrarse como si fuera algo sustancial, debe apelarse a coordenadas que soporten toda ocasionalidad. Todo contexto está tomado y limitado por un contexto mayor, que garantiza un mínimo de interacción y mutuo entendimiento. Ese entorno de relaciones, conducido hasta la díada adulto-bebé, no necesita pensarse como un lenguaje primigenio e irreconocible. Ciertos empleos parasitarios, que tendrían que ver con un fracaso comunicativo (pero que definirían conceptualmente cualquier posibilidad de uso exitoso), sólo están testimoniados como logros ulteriores de la adquisición lingüística. El desarrollo de la mente impide la mentira en el primer momento. Los niños con el síndrome de Williams son conversadores competentes que no logran manejar dobles sentidos. Cronología y psicopatología parecen, por lo tanto, favorecer cierto desdoblamiento entre el empleo ordinario y el parasitario.

Al condenar (en la obra de Platón) cómo el discurso oral es valorado por encima de la letra escrita, Derrida parece obviar que en la postura denunciada se rescata, sobre todo, la oportunidad de dialogar, la circunstancia en la que nace la palabra y que apunta hacia un mundo compartido que las convenciones no pueden llevar alegremente adonde quieran. Este contexto matricial de interacciones contiene en su seno los contextos culturales y epocales, el Sentido del sentido desde el que poner en duda este o aquel sentido o interpretación, porque sólo sobre un idioma compartido puede haber malentendidos. Hay un idioma humano que es social y es el contexto de todo contexto de interpretación. Y ese contexto de interpretación surge y se consolida $-\mathrm{y}$ deconstruye y reconstruye, se enmienda y se rectifica - siempre desde el diálogo, la interacción significada. Muy pronto alegará el simpatizante derrideano que las convenciones, en 
cuanto producto de interlocuciones, no implican un suelo firme, sino una estabilidad fluyente. Pero debe enfrentar el hecho de que las interacciones y las significaciones más fundamentales, para llegar a plasmarse en convenciones con los semejantes, dependen de los contactos intersubjetivos, del trato con los objetos, contactos y trato que tienen la forma de un cuerpo específico (en la acepción: species-specific), que anda erguido (aunque comienza en cuatro patas), que toca y explora el mundo con dos manos, nunca más que dos, y que las oportunidades conductuales que habilita esta figura bípeda con pulgar oponible condicionan universalmente nuestra familiaridad con los objetos, la capacidad de asirlos, nuestra construcción del espacio inmediato, forjado desde los movimientos, y las transacciones físico-afectivas con los prójimos, que tienen, por su parte, un cuerpo semejante. La relación del individuo con el mundo está mediada por el cuerpo y la pluralidad de cuerpos. De ello será posible una semiótica codificada con arreglo a ciertas pautas práxicas que sedimentan en nuestras certezas. Los empleos parasitarios del lenguaje tienen que apoyarse en significaciones previas que vuelen muy cerca de este nivel de certezas. Toda verdad textual y diseminación de los sentidos regresa infaliblemente a este nivel para recuperar su centro. Sólo para el lenguaje adulto lo parasitario es co-constitutivo, pero las interpretaciones de tipo más liberal siempre están limitadas por un marco de categorías hormantes radicadas en la corporalidad. La verdad, textual o contextual, tiene una plataforma con nodos poco permeables a interpretación. Los molinos de viento pueden parecer gigantes, pero en la realidad - como defiende Peirce - algo resiste y pone frente el más quijote la dureza del muro de piedra.

Los estudios sobre ontogénesis respaldan, desde la evidencia empírica, que los procesos subjetivos de semiosis no están dados por oposiciones primigenias ni por la deriva semoviente de significantes, factores contrarios a la hipótesis de convenciones o certezas interculturales y trans-epocales, ni se hallan determinados por un ejercicio que, referencial, procese las experiencias infantiles (preverbales) en la criba de lo verdadero/falso. Antes de la palabra, el gesto y la expresión del rostro se conjugan para una semiótica carente de verdad, y la segmentación de eventos habituales antes mencionada, en la que un elemento se destaca del conjunto en representación del todo o del faltante (semiosis por suspensión — Rivière, 2003 [1984]; Rivière y Español, 2003), lleva a pensar que la primera significación no responde a un criterio opositivo, como el de los estructuralistas (incluso cuando se concediera que el lenguaje abarque toda variedad de signos), sino a un criterio de figura-contexto o de fondo-figura (que no es apropiado homologar con un contraste de unidades al mismo nivel, lo que sería tomar la relación entre elementos de un conjunto por la relación entre cierto elemento y el conjunto como tal). La asociación y la anticipación del niño, como procesos mentales necesarios, desaguan en la semiosis, que potencia la eficacia previa en las interacciones y la también anterior comprensión del entorno. Cuando más tarde la composición de signos permita hacer enunciados y exista en el niño madurez intelectual como para reflexionar sobre sus creencias sive expectativas, los procesos de semiosis se intersecarán con la verdad, pero este cruce no es indispensable para que el signo realice como tal un primer tipo de trabajo. 


\section{CONCLUSIONES}

Durante buena parte del siglo XX, la semiótica ha sido pensada otorgando al lenguaje unas prerrogativas con las que éste ha detentado un rol central o normativo. Por su vinculación con la verdad, esta llegó a perimetrar el campo de los estudios semióticos. Lo hizo por vía de una semántica de fundamentos lógicos, dando lugar a un logicismo de la significación y a un maridaje con la epistemología; lo hizo también por una alternativa que, fundada en la lingüística de corte saussureano, por ende bajo un concepto diferente de lenguaje, comenzando por la idea de lengua, llegaría a una relativa absolutización de lo lingüístico sobre toda faceta cultural o del conocimiento, y a replantear la condición humana en general (esencialmente denotada por significantes o sentido) y la idea de verdad. De modo respectivo, hemos denominado a estas opciones perspectiva referencialista, semiótica afirmada en la proposición y en relaciones lógico-lingüísticas, y perspectiva significacionista, atenta al mecanismo de significar. Una tercera alternativa, en realidad parte de la segunda y que en nuestro periplo sólo hemos rozado al paso, sorteó con habilidad la trampa lingüistizadora, pero anudaba los procesos perceptivo-inferencial a los hechos semióticos, arribando también a un desenlace de tipo epistemizante, para la mayor gloria de Peirce. Peirce había visto los inconvenientes del lenguaje y la proposición, pero no resignó el formato de inferencia o inducción (interpretante emocional y energético); al mismo tiempo, fue quien proporcionó un escape de este esquema en la noción de hábito, que resuena a su vez en la certeza preveritativa y las rutinas preverbales.

De una u otra manera, la semiótica quedaba inextricablemente vinculada a la verdad, dentro del horizonte de la cognición y del lenguaje. La psicología del desarrollo, en su investigación de las habilidades significativas del niño pequeño y de sus condiciones posibilitantes, nos muestra a los signos como auxilios de una interacción preverdadera, una pragmática en la que se forja la semántica antes de cualquier sintaxis, un territorio sin verdad, preintelectual, antepredicativo, que precede a la proposición y le aporta sus contenidos. Los primeros signos no son del lenguaje ni están asociados al saber. Las tradiciones que fueron examinadas no negaron la existencia de signos gestuales preverbales, pero o les restaron importancia, por no conformarse a la proposición, o los asimilaron (y a toda otra forma de semiosis) a una forma de estructura hecha de puras diferencias. Las utilidades no epistémicas de estos primeros signos impugnan que la semiosis deba definirse en términos de la verdad.

Respecto del lenguaje como heurístico y explicación, ni recubre todo el terreno de los signos, ni estos se ajustan a un dispositivo de elementos oponibles e insignificantes. El sentido no nace de un aparato formal o simbólico que espera al individuo y lo sujeta, sino de un proceso activo que lleva de las asociaciones a la representación de una cosa por otra. Respecto de la verdad, no existe la necesidad de vincularla, en un primer momento, a la semiosis. Corresponde postergar este entrecruzamiento hasta que la composición de 
signos permita los enunciados $\mathrm{y}$, por su intermedio, proposicionalizar los pensamientos, lo que no sucede sino hasta después de que la significación se haya consolidado como medio para o instrumento de uso. En la etapa pragmática inicial es posible entender que hay un divorcio conceptual entre semiosis y conocimiento.

Es la psicología quien, simultáneamente, nos pone en la huella de que la interpretación, toda interpretabilidad, descansa en una realidad co-construida. Cuando aparezca para el niño, con la edad, el juego de ficción, que exigirá un desasimiento de la realidad, este solo comprenderá una parte de la realidad, no su totalidad, y el amparo incondicional de las certezas subyacentes (lo que deja entender que la interpretación está invariablemente atenta a constricciones que la exceden y preceden, de las cuales puede por supuesto tomar una o más licencias, pero nunca emanciparse quemando las naves). Cuerpo y acción, antecedentes informantes de la significación y del lenguaje, son a la vez las alas y el aire que surca la imaginación en vuelo.

Semiosis y cognición del mundo no son un mismo proceso o territorio. Semiosis y lógica o conocimiento abstracto no son dimensiones coextensivas. Antes de la fecunda fraternización de estas dos facultades, saber y significar, los hechos de interpretación y producción semiótica se añaden al mundo del niño como facilitadores de la socialización y la empatía (de donde prestan asistencia a las actividades práxicas en la constitución de las certezas que dan entidad a cosas y a personas). Según ello, la semiótica qua disciplina ha de ser definida en prescindencia de constreñimientos epistémicos. Si la verdad puede tomarse como condicionamiento suficiente del fenómeno semiótico, éste sucede ser, por lo contrario, condición de tipo necesaria, pero insuficiente. De modo que la ecuación no se sostiene, y la semiótica debe pensarse desde la sustituyente fórmula de la que hemos partido, aliquid [quod stat] pro aliquo.

\section{REFERENCIAS BIBLIOGRÁFICAS}

Austin, J. L. (1975 [1962]). Ensayos filosóficos. Madrid: Revista de Occidente.

Ávila CAÑAMARES, I. (2014). “Atención, referencia e inescrutabilidad". Estudios de Filosofía 50, 31-50.

BARTHES, R. (1967). Système de la mode. Paris: Seuil.

Beuchot, M. (1987). Aspectos históricos de la semiótica y la filosofía del lenguaje. México DF: Universidad Autónoma de México.

CAmpBell, J. (2002). Reference and Consciousness. Oxford: Oxford University Press.

DAVIDSON, D. (1973). "Radical Interpretation". Dialectica 27, 314-28.

DERRIDA, J. (1967a). La voix et le phénoméne: introduction au problème du signe dans la phénoménologie de Husserl. Paris: PUF. (1967b). De la grammatologie. Paris: Minuit. (1967c). L'écriture et la différence. Paris: Seuil.

ECO, U. (1980 [1973]). Segno. Mondadori: Milano. 
(1990 [1984]) Semiología y filosofía del lenguaje. Barcelona: Lumen.

(1999 [1997]). Kant y el ornitorrinco. Buenos Aires: Sudamericana.

Escudero-Sanz, A.; CARranZa-CARnicero, J. A. y HuÉSCAR-HernándeZ, E. (2013).

"Aparición y desarrollo de la atención conjunta en la infancia". Anales de psicología 29.2, 404-412. Disponible en línea: http://dx.doi.org/10.6018/ analesps.29.2.136871 [04/05/2021].

ESPAÑOL, S., ED. (2014). Psicología de la música y del desarrollo. Una exploración interdisciplinaria sobre la musicalidad humana. Buenos Aires: Paidós.

FreGe, G. (1892). "Über sinn und Bedeutung". Zeitschrift für Philosophie und philosophische Kritik 100, 25-50.

Goodman, N. (1968). Languages of Art. Indianapolis: Bobbs-Merrill.

GRICE, H. P. (1975). "Logic and conversation". En Studies in the Ways of Words, H. P. Grice, 22-40. Cambridge, MA: Harvard University Press.

KARMiloff, K. Y KARMiloff-SMIth, A. (2001). Hacia el lenguaje. Madrid: Morata.

Leduc, K.; Williams, S.; Gomez-Garibello, C. \& Talwar, V. (2017). "The contributions of mental state understanding and executive functioning to preschool-aged children's lie-telling". British Journal of Developmental Psychology 35.2, 288-302. Disponible en línea: https://doi.org/10.1111/ bjdp.12163 [04/05/2021].

LÉVI-STRAuss, C. (1970). El pensamiento salvaje. México: FCE.

LOCKE, J. (2005 [1690]). Ensayo sobre el entendimiento humano. México DF: FCE.

MiLL, J. S. (1905 [1843]). A System of logic. London: Routledge.

Navarro Reyes, J. (2010). Cómo hacer filosofía con palabras. A propósito del desencuentro entre Searle y Derrida. Madrid: FCE.

Nelson, K. (1996). Language in Cognitive Development: The Emergence of the Mediated Mind. New York: Cambridge University Press.

NöTH, W. (2000). Handbuch der Semiotik. Sttutgart-Weimar: Metzler.

QuINE, W. V. O. (1960). Word and Object. Cambridge, MA: MIT Press.

(1988 [1974]). Las raices de la referencia. Madrid: Revista de Occidente.

PeIRCE, CH. S. (1972). The Essential Writings. New York: Harper Row.

PINBORG, J. (1972). Logik und Semantik im Mittelalter. Sttutgart: Frommann.

Rivière, Á. (2003 [1984]). "Acción e interacción en el origen del símbolo". En Ángel Rivière. Obras Escogidas, M. Belinchón, A. Rosa, M. Sotillo e I. Marichalar (eds.), vol. II, 77-108. Madrid: Panamericana.

Rivière, Á. Y ESPAÑOL, S. (2003). "La suspensión como mecanismo de creación semiótica". Estudios de Psicología 23.4, 261-275.

RodrígueZ, F. G. (2011). "Afanes incumplidos del sueño estructuralista. Lenguaje, lógica y formalización en Lévi-Strauss y Lacan”. En El Estructuralismo en sus márgenes. Ensayos sobre criticos y disidentes: Althusser, Deleuze, Foucault, Lacan y Ricoeur, F. G. Rodríguez y M. Vallejo (eds.), 15-77. Buenos Aires: Del Signo. 
RODRÍGUEZ, F. G. Y ESPAÑOL, S. (2019). "Las funciones comunicativas durante la etapa pregramatical: un estudio de caso". Infancia y Aprendizaje: Journal for the Study of Education and Development 42.2, 435-456.

Saussure, F. DE (1995 [1916]). Cours de linguistique générale. Publiée par Charles Bailly et Albert Séchehaye avec la collaboration de Albert Riedlinger. Édition critique par Tullio de Mauro. Postface de Louis-Jean Calvet. Paris: Payot et Rivages.

SAZBÓn, J. (1976). "Significación del saussurismo". En Saussure y los fundamentos de la lingüistica, J. Sazbón, 9-54. Buenos Aires: Centro Editor de América Latina.

Searle, J. (1969). Speech acts. An essay in the philosophy of language. Cambridge: Cambridge University Press.

Short, T. L. (2007). Peirce's Theory of Signs. Cambridge: Cambridge University Press. Strawson, P. F. (1950). “On Referring”. Mind 59, 320-344.

Trevarthen, C. (1980). "The Foundations of Intersubjectivity: Development of Interpersonal and Cooperative Understanding in Infants". En The Social Foundation of Language and Thought, D. R. Olson (ed.), 316-342. New York: Norton.

VATER, H. (2005). Referenzlinguistik. München: Wilhelm Fink

Wittgenstein, L. (1963 [1922]). Tractatus Logico-Philosophicus. Frankfurt am Main: Suhrkamp

(1969). Über gewissheit. Oxford: Blackwell. (1988 [1953]). Investigaciones filosóficas. Barcelona: Altaya.

Wolff, P. H. (1987). The Development of Behavioral States and the Expression of Emotions in Early Infancy: New proposals for Investigation. Chicago: University of Chicago Press.

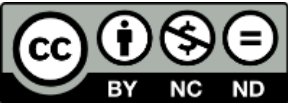

This work is licensed under a Creative Commons AttributionNonCommercial-NoDerivatives 4.0 International (CC BY-NC-ND).

Fecha de recepción: 19/01/2021

Fecha de aceptación: 14/05/2021 